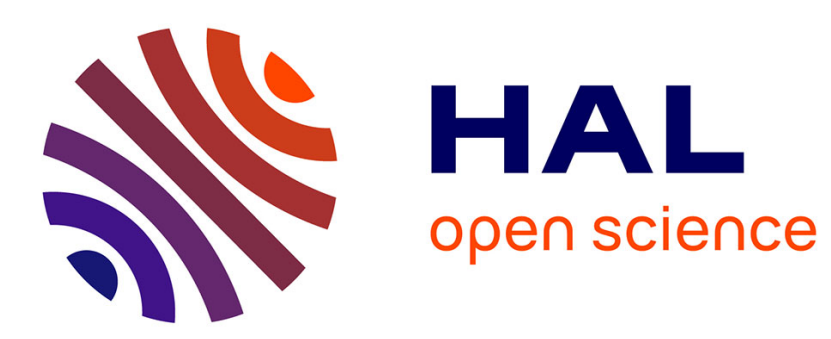

\title{
Entre art et science, l'histoire à l'Institut d'urbanisme de l'Université de Paris (1919-1971) \\ Grégory Busquet, Claire Carriou
}

\section{To cite this version:}

Grégory Busquet, Claire Carriou. Entre art et science, l'histoire à l'Institut d'urbanisme de l'Université de Paris (1919-1971). Espaces et sociétés (Paris, France), 2007, 130, pp.57-70. 10.3917/esp.130.0057 . halshs-00458184

\section{HAL Id: halshs-00458184 \\ https://shs.hal.science/halshs-00458184}

Submitted on 9 Nov 2018

HAL is a multi-disciplinary open access archive for the deposit and dissemination of scientific research documents, whether they are published or not. The documents may come from teaching and research institutions in France or abroad, or from public or private research centers.
L'archive ouverte pluridisciplinaire HAL, est destinée au dépôt et à la diffusion de documents scientifiques de niveau recherche, publiés ou non, émanant des établissements d'enseignement et de recherche français ou étrangers, des laboratoires publics ou privés. 


\title{
Entre art et science, l'histoire à l'Institut d'urbanisme de l'université de Paris (1919-1971)
}

\author{
Grégory Busquet \\ Claire Carriou ${ }^{1}$
}

\section{$\mathrm{S}_{\mathrm{i}}$} la mobilisation du passé dans la pratique urbanistique n'est pas affaire récente, son statut diffère largement selon les époques. On peut saisir cette évolution à travers l'étude de l'enseignement à l'Institut d'urbanisme de l'université de Paris (IUUP), de sa fondation à sa dissolution ${ }^{2}$. Premier et longtemps unique centre de formation des urbanistes en France, l'IUUP reste pendant plusieurs décennies un lieu de référence de la culture urbanistique, même si son autorité tend à décliner au cours des trente glorieuses avec la

Grégory Busquet, doctorant à l'Institut d'urbanisme de Paris, Centre de recherche sur l'habitat - LOUEST (UMR 7145)

gregory.busquet@paris-valdeseine.archi.fr

Claire Carriou, doctorante à l'Institut d'urbanisme de Paris, Centre de recherche sur l'habitat - LOUEST (UMR 7145)

claire.carriou@paris-valdeseine.archi.fr

1. Les auteurs tiennent à remercier Laurent Coudroy de Lille, maitre de conférences à l'Institut d'urbanisme de Paris, pour les avoir orientés dans cette recherche.

2. L'École des hautes études urbaines fondée à Paris en 1919 devient Institut d'urbanisme de l'université de Paris en 1924. Rattaché à l'université de Paris-Dauphine de 1969 à 1971, l'Institut est ensuite refondé à Créteil (université de Paris XII) sous le nom d'Institut d'urbanisme de Paris. 
création de l'atelier d'urbanisme du Séminaire Tony Garnier à l'École des Beaux-Arts (1961), puis celle d'autres instituts à Paris ou en province, comme l'Institut français d'urbanisme en 1969. Voué à former les praticiens chargés de réaliser des plans et des personnels administratifs - sa création, sous l'appellation d'École des hautes études urbaines (EHEU), suit le vote de la première loi d'urbanisme en France en 1919 -, cet Institut constitue aussi l'un des pôles d'émergence d'une tradition d'études urbaines. L'enseignement s'y construit sur l'ambition d'allier art et science urbaine, avec un recours central à l'histoire. Visant un but autant scientifique qu'édilitaire, son programme initial entend saisir « l'agglomération urbaine envisagée comme un organisme vivant qui évolue dans le temps et dans l'espace » (La vie urbaine $\mathrm{n}^{\circ} 1-2,1919$, avant-propos). Enseignement et programme historiques sont largement dus à Marcel Poëte (1866-1950), archiviste-paléographe issu de l'École des Chartes, cofondateur et directeur de l'EHEU, qui donne ses cours de 1919 à 1937. En prenant la direction de l'Institut en 1942, l'historien de l'art Pierre Lavedan (1885-1982) va y développer une nouvelle approche de l'histoire.

Des monographies ont déjà été consacrées à Marcel Poëte (Donatella Calabi), Pierre Lavedan (Isabelle Grudet) et à l'Institut lui-même (Rémi Baudouï). Il s'agit ici de mettre en perspective, pour l'ensemble de la période envisagée (1919-1971), les apports respectifs de ces deux historiens de l'IUUP. Leurs conceptions différentes de l'usage de l'histoire s'analysent en parallèle avec les évolutions mêmes de la discipline urbanistique. Ces conceptions ont ainsi des effets sur les approches de la planification et sur la recherche, comme en témoignent les travaux de leurs étudiants, leurs « thèses ».

\section{Marcel Pö̈te, La fonction heuristiQue de « L'Évolution URbaine » DANS LA PLANIFICATION}

Dans l'entre-deux guerres, l'histoire est une discipline fortement présente à l'IUUP. Absente de l'École nationale d'administration municipale, nouvelle filière créée en 1924 destinée à la formation des agents administratifs, elle constitue en revanche l'un des cours fondamentaux de l'enseignement d'urbanisme, qui met côte à côte enseignements à tendances théoriques et pratiques ${ }^{3}$. L'histoire enseignée par Poëte a en effet vocation de nourrir l' « art urbain », soit la mise en forme des plans d'aménagement.

Pour autant, son cours n'est pas un cours d'histoire au sens universitaire. Les temporalités abordées ne se limitent pas au temps passé. Le choix même

3. Les quatre cours originaux sont l'évolution des villes, l'organisation sociale des villes, l'organisation administrative des villes, et enfin l'art urbain auxquels s'ajoutera plus tard l'organisation économique. 
de son intitulé « Évolution des villes » est à cet égard révélateur. Il s'agit davantage d'envisager l'étude des villes dans un horizon chronologique large embrassant à la fois passé, présent et avenir, plutôt que de travailler à la reconstitution raisonnée de leur état antérieur. C'est leur position dans un cycle « vital », comme l'indique le terme « évolution », qui est étudiée. Les villes sont vues comme des êtres collectifs, des organismes, qui naissent puis meurent : «Étant donné une ville, il faut se demander quel est son passé, son âge véritable, à quel point de son évolution elle est parvenue. S'agit-il d'une ville autrefois considérable et aujourd'hui déchue, ou d'une ville en plein développement ?» (Poëte, 1919-1920, nº 1:3). Leur analyse dans la longue durée permet de dégager ce qui constitue leur identité et leur dynamisme urbain, économique, social, politique. La continuité importe davantage que les ruptures : plus qu'à la chronologie, Poëte s'attache au système des causes. Par le croisement d'exemples de villes dans l'histoire, il tend à dégager quelques-uns des principes généraux, à peine qualifiés de « lois », qui conditionnent selon lui le développement des villes : le cadre géographique, les voies de circulation et d'échange. Aussi hésite-t-il dans la façon de présenter au mieux ses approches. Penchant d'abord pour un exposé chronologique sur les grands types de villes au cours des siècles, il s'oriente dans les années 1920 vers une analyse mêlant divers exemples éclairants du point de vue méthodologique (Poëte, 1919-1920, 1926-1927).

Néanmoins, il n'ignore pas les références du monde de l'histoire universitaire (Calabi, 1998). Fortement marqué par l'histoire méthodique, Poëte a lu également les historiens de la nouvelle génération, qui formeront bientôt l'École des Annales. Son analyse des continuités de la ville n'est pas sans rappeler leur projet de réaliser des grandes synthèses. Il s'en rapproche également par son refus du déterminisme historique des conceptions plus classiques de l'histoire : le recours à la notion « d'élan vital » de Bergson traduit cette volonté de privilégier une approche possibiliste de l'enchaînement des faits historiques (Calabi, op. cit.). Enfin, dans le travail de Marcel Poëte, apparaît l'expression des nouveaux courants de recherche en histoire, où s'instaure un dialogue avec la géographie. Construite sur une approche monographique et accordant une place prédominante à l'étude du site, l'étude d'évolution urbaine s'appuie, parallèlement à un dépouillement archivistique, sur une observation des formes (Poëte, 1926-1927 $\mathrm{n}^{\circ} 1: 4$ ). Mais ces proximités sont de surface. Poëte est largement critiqué dans les Annales - Lucien Febvre lui reproche le caractère trop général de ses études - et demeure en marge de l'université (Calabi, op. cit., 83 ; Claude, 2006).

Son enseignement à l'IuUP, loin d'engager à la seule recherche historique, a pour finalité de fonder la réalisation des Plans d'aménagement, d'embellissement et d'extension (PAEE) - rendus obligatoires depuis 1919 - sur la prise en compte du temps long. Le PAEE conduit en effet, non plus seulement à aménager l'existant, mais à prévoir l'extension future de la ville. L'objet de 
son cours, repris en 1929 dans son ouvrage Introduction à l'urbanisme, est l'apprentissage de la première étape de la méthode d'aménagement des villes. Celle-ci concerne l'analyse " des conditions et des manifestations d'existence et de développement de la cité dont il s'agit de dresser le plan ». « La deuxième étape consiste à dégager de l'étude précédente tout ce qui est susceptible de conditionner l'avenir de cette cité. Il s'agit de discerner dans le présent les germes de l'avenir. » La troisième étape est la réalisation du plan à partir de ces données (Poëte, 1919, $\mathrm{n}^{\circ} 1: 3$ ).

Ces conceptions le rapprochent, dès l'avant-guerre, de celles des architectes et personnalités impliquées dans les réflexions sur l'aménagement urbain. Initiateur d'un cours sur l'histoire de Paris dès 1906 à la Bibliothèque de la ville de Paris où il est conservateur depuis 1903, il occupe le poste de secrétaire général de la Commission du vieux Paris à partir 1913. Il est également lié au Musée social, où se formalisent les premières idées sur l'urbanisme. Il côtoie les fondateurs et membres de la future Société française des urbanistes (SFU) officialisée sous ce nom en 1921, parmi lesquels les architectes Louis Bonnier (1856-1946), Donat-Alfred Agache (1875-1959), Léon Jaussely (1875-1933). Poëte partage avec eux un intérêt pour la « morphologie urbaine » envisagée comme expression de la vie sociale ainsi qu'un intérêt pour le temps long de la ville. Attentifs, de par leur formation à l'École de beaux-arts, aux formes architecturales et urbaines historiques, ces architectes sont aussi familiers des conceptions de P. Geddes, biologiste anglais, qui préconise d'appréhender la ville selon une approche évolutionniste (Bruant, 2001, 136 ; Puyo, 2001, 128). Dès 1915, Jaussely, dans sa contribution à un ouvrage considéré comme le premier manuel français d'urbanisme, propose d'asseoir la réalisation du plan sur l'établissement d'un programme prenant en compte les questions relatives au "passé de l'agglomération, ses conditions géographiques, économiques et sociales, son état actuel et son avenir probable » (Agache et al., 1915, 13). Avec son enseignement d'évolution des villes, Poëte contribue ainsi à consolider d'un point de vue théorique une méthode d'aménagement urbain fondée sur l'observation du milieu.

\section{LE PASSÉ, LE PRÉSENT ET L'AVENIR DANS LA RÉALISATION DU PLAN (1919-1939)}

La première équipe enseignante fait corps avec ce projet pédagogique. Léon Jaussely est chargé du cours d'art urbain, en symbiose avec la perspective dessinée par Poëte (Jaussely, $1920 n^{\circ} 1:$ 6). La méthode proposée par ce dernier apparaît bien comme une ligne forte de l'enseignement de l'urbanisme à l'Institut. C'est dans l'exercice consistant à faire systématiquement précéder la planification d'une étude préalable du site - dont l'étude d'évolution devient un passage obligé - que cet héritage se manifeste le plus clairement. Cet exercice devient un classique de la formation à l'IUUP. Dans la 
revue La vie urbaine, « organe de l'enseignement » dès la fin 1919 et qui devient dans les années 1930 un outil pédagogique, de nombreux articles monographiques s'inspirent de cette approche et intègrent, de manière quasi systématique, l'histoire du site. C'est de cette même méthode que Gaston Bardet (1907-1989), héritier intellectuel de Poëte et assistant à l'IuUP à la fin des années 1930, s'inspirera pour théoriser ce qu'il appelle le « diagnostic urbain ». Celui-ci, intégrant le recours à l'histoire, vise à fonder la planification urbaine sur un « langage dynamique qui permette [à la ville] de s'exprimer dans la durée » (Bardet, 1948 [1941], 13).

La diffusion et la vulgarisation de cette approche s'accompagnent néanmoins d'une dénaturation des objectifs énoncés par Marcel Poëte dans son cours. Dans les études monographiques parues dans La vie urbaine, l'exposé historique fait certes figure de préalable incontournable, mais il tend à s'autonomiser de la composition du plan. Schémas et idées permettant de tirer du passé les «germes » de l'avenir qui font défaut. Dans les faits, l'entre-deux guerres voit progressivement s'exprimer des divergences de conception quant aux dynamiques de l'évolution urbaine. Attaché à une vision vitaliste de la ville, Poëte a tendance à réduire l'aménagement urbain à un simple rôle d'accompagnement du mouvement naturel de la cité (Baudouï, 1988b), - « mouvement humain, de vie continue, évoluant » (Poëte, 1926-1927, $\mathrm{n}^{\circ} 8: 1$ ). Praticiens et théoriciens de l'urbanisme, de leur côté, affirment plus nettement la légitimité de l'intervention humaine et lui reprochent sa vision fataliste de l'histoire (Rosenthal, 1918, 85) voire son conservatisme (Bruant, op. cit., 147). Cette critique n'est-elle pas, par ailleurs, le signe d'une affirmation de compétences proprement pratiques dans la planification ? Le contexte, marqué par une multiplication des projets d'aménagement et une certaine structuration du métier d'urbaniste (Claude, op. cit), est propice à un réajustement des positions et des méthodes. Exemple de cette tendance, l'équipe Jacques Gréber (1885-1962), Henri Prost (1874-1959), Louis Bonnier, qui succède à Jaussely en 1927 dans l'enseignement de l'art urbain, propose de mobiliser l'histoire selon des modalités différentes. Ainsi l'ouvrage de Jacques Gréber sur l'aménagement de Marseille (Gréber, 1933), considéré comme « le principal et le plus représentatif de l'enseignement professé à Paris »(La Vie urbaine, 1933, n $17: 314)$, n'accorde qu'une place réduite à l'exposé historique écrit (une page sur cent onze). Un tel exposé risquerait, en effet, selon l'auteur, de « tomber dans le domaine du rêve et de la littérature » (Gréber, op. cit., 3). Le passé est désormais appréhendé par ses empreintes marquant la physionomie urbaine. Gréber propose une méthode pratique guidée par le souci essentiel de se confronter au terrain (ibid., 321). Dans cette perspective, le recours à l'image, plus approprié au travail de composition, prend le pas sur l'écrit pour argumenter la direction d'un tracé. Si l'histoire demeure donc utile à la planification, elle tend peu à peu à l'être davantage par l'héritage formel que par l'étude d'évolution urbaine. 


\section{Pierre LaVedan À L'IUUP : POUR Une histoire ET UN ReSPeCT DES FORMES URBAINES}

Les rapports entre histoire et urbanisme se recomposent après 1945. L'histoire tend à s'isoler dans le projet pédagogique, en écho à l'évolution de la demande sociale en matière d'urbanisme. Les impératifs de productivité et les méthodes volontaires qui marquent la Reconstruction et les dites « trente glorieuses » s'accordent mal avec un respect de l'histoire. L'IUUP résiste cependant aux théories de l'urbanisme moderne qui vise à faire table-rase du passé. Mais en dépit des efforts de Pierre Lavedan, le cours d'évolution des villes, qu'il reprend en 1940, au moment même où il obtient la chaire d'histoire de l'architecture à l'École des Beaux-Arts, perd sa position privilégiée pour s'intégrer dans le cursus au même titre que les autres sciences humaines. Par ailleurs, son approche de l'histoire accompagne la reformulation de l'urbanisme, en accordant, à la différence de Marcel Poëte, un rôle central à l'action de l'homme dans l'histoire des villes et en proposant un autre mode d'intégration de l'histoire à la planification.

La construction chronologique de son cours et l'importance des études de cas rappellent son prédécesseur, mais il s'en écarte dans ses enseignements et publications sur plusieurs points. Lavedan refuse d'abord de faire de l'urbanisme une « science », le considérant comme un «art» (Lavedan, 1975). À l'Institut, cet intérêt pour l'art l'amène à consacrer une grande partie de ses cours à l'histoire de l'architecture et des formes urbaines. Le projet d'étude de l'évolution des villes perçues comme des êtres organiques, est oublié, rendant caduc le terme d' « évolution » attaché à retranscrire leur caractère dynamique. Là où Poëte parle d'évolution, Lavedan parle de développement et critique à demi-mot ce dernier : « La ville, dit-on, est un être vivant et, comme tous les êtres vivants, elle naît, elle grandit, elle meurt [...]. La comparaison, que j'ai longtemps acceptée moi-même [...], me paraît aujourd'hui inacceptable [...]. La ville, au contraire, peut rajeunir ou même ressusciter. Surtout, alors que l'homme commence par être enfant, beaucoup de villes n'ont pas eu d'enfance, sont apparues du premier coup en pleine force $[\ldots]$ : ce sont les villes créées, dont les théories fatalistes ignorent l'existence et nient même implicitement le principe » (Lavedan, 1959 [1937], 13-144).

L'étude des plans de ville, se référant directement à l'action des hommes (princes, architectes...), l'amène à aborder la ville comme une création individuelle, alors que Poëte la percevait comme évoluant par l'action de l'être collectif qu'elle représentait à ses yeux. Lavedan fait peu de cas des «villes spontanées ». Il se situe donc dans l'histoire de l'urbanisme et des formes,

4. Ces considérations, datant de la première édition de La géographie des villes en 1937, sont reprises dans l'introduction de son cours à l'IUUP (années 1948-1949). 
qu'il qualifie tantôt d'histoire " des créations urbaines », tantôt d'histoire de " l'architecture urbaine », en se fondant sur les travaux des géographes (Pinon, 1996, 115). Alors que Poëte couvrait, par son histoire urbaine, des "préoccupations "sociologiques, géographiques et économiques" », Lavedan les utilise «pour expliquer l'“architecture urbaine"» (ibid., 112). L'intitulé de son cours, repris par son assistante Jeanne Hugueney, reflète largement cette évolution. Des années 1940 aux années 1950, on passe d'« évolution des villes »- professé par Lavedan et Pierre Lelièvre, bibliothécaire et historien chartiste - à « évolution des villes et histoire de l'urbanisme » - Lavedan et Hugueney. Puis le cours s'intitule « histoire de l'urbanisme et évolution économique des villes » - Lavedan, Hugueney - et enfin simplement « histoire de l'urbanisme » dans les années 1960, cours assuré par Hugueney et Gilbert Picard, archéologue spécialiste de l'Antiquité. Le terme « évolution » disparaît et l'intérêt pour l'urbanisme seul est de plus en plus mis en avant ${ }^{5}$. Lavedan imprime également sa marque à la ligne éditoriale de $\mathrm{La}$ vie urbaine, qu'il dirige à partir de 1950. Les articles à visée historique y sont dorénavant presque exclusivement consacrés à l'histoire des formes et de l'architecture, ainsi qu'aux monuments historiques.

Lavedan intègre l'histoire à la planification par les formes architecturales et urbaines. L'histoire doit servir à la préservation des monuments du passé, par un repérage systématique des constructions à conserver dans un souci patrimonial et esthétique. Elle doit servir également à la projection, en fournissant ce qu'Isabelle Grudet nomme des « archétypes d'action » formels (Grudet, 2005b, 171). Le but n'est pas de fournir des modèles reproductibles hérités du passé mais de trouver des solutions aux problèmes contemporains de la ville en adaptant les expériences du passé à la situation contemporaine. Dès 1926, il s'agit de : «faire de l'archéologie, non pas en spéculatifs, mais “en gens qui demandent au passé tout ce qu'il pourrait donner au présent et surtout à l'avenir"» (Lavedan, 1926 : avant-propos). L'histoire des villes est appréhendée par l'étude de l'évolution des plans (réduits aux réseaux viaires) et de l'iconographie, dont la connaissance aide à la réalisation d'un urbanisme idéal et viable. L'objet est donc de constituer une « doctrine » utile aux « architectes urbanistes » (ibid.), construite sur l'analyse des plans du passé. Ce recours systématique aux expériences formelles du passé dans la planification a constitué le principal motif de critique de sa méthode de la part des historiens de l'École des Annales. Ces derniers soulignent son « amateurisme » et ne comprennent ni son utilisation des sources iconographiques ni son histoire des villes ne prenant pas en compte leur aspect social (Calabi, op. cit.).

5. Voir l'article d'Isabelle Grudet dans ce numéro. 


\section{VERS L'AUTONOMISATION DE L'HISTOIRE DES VILLES COMME DISCIPLINE DE RECHERCHE}

Les conceptions de Lavedan ne suffisent pas à surmonter le clivage désormais affirmé entre théorie et pratique, dont on perçoit les prémices dès l'entre-deux-guerres. Le cursus de l'IuUP est en effet réorganisé, divisé entre cours techniques (Robert Auzelle, Jacques Gréber, Pierre Rémaury...) et enseignements généraux, plus universitaires avec le droit, la géographie et bien sûr l'histoire. Le cours intégrateur est désormais celui consacré à la " théorie générale de l'urbanisme » professé par Robert Auzelle (1913-1983) de 1956 à $1968^{6}$. Ce cours vise la synthèse entre théorie et pratique et prend place dans les enseignements dits techniques. Auzelle, autre grande figure de l'IUUP de l'après-guerre s'inscrit dans la filiation de Jaussely, Prost et Gréber - son directeur de thèse. Certes il accorde une place non négligeable dans l'enseignement de sa méthode urbanistique à l'histoire, «mémoire d'un devenir » qui « apporte des éléments d'information par lesquels s'expliquent ou se justifient telles formes actuelles de l'occupation d'un territoire». L'urbaniste doit en tenir compte pour "adapter le cadre aux exigences du présent et surtout, aux libertés du futur » (Auzelle, 1967, 60). Malgré l'attention portée aux conceptions de Poëte et de Barde, Auzelle, urbaniste en chef du ministère de la Reconstruction après-guerre, demeure ainsi le représentant de l'aspect « technique » de l'urbanisme à l'Institut, prenant l'ascendant sur l'histoire des formes urbaines de son collègue Lavedan.

On observe, ainsi, une autonomisation relative à l'histoire. Elle n'est plus la discipline transversale de l'urbanisme et se consacre dorénavant à la seule recherche sur le passé. Cette tendance est perceptible dès les années 1950 dans La vie urbaine. Les articles d'histoire de l'urbanisme ou de l'art ne donnent pas d'indication claire quant à leur apport possible à la planification. Lavedan et Jeanne Hugueney détiennent dorénavant le quasi-monopole de leur rédaction, ce qui diffère de la période avant-guerre pendant laquelle les articles d'histoire n'étaient pas dus au seul Poëte. En 1953, par exemple, un double numéro consacré à Haussmann (La vie urbaine, 1953, n 3-4), dans lequel Lavedan rédige la quasi-intégralité des articles, aux côtés d'Hugueney.

Bien plus, l'histoire est mise l'écart dans le projet pédagogique global de l'Institut. C'est paradoxalement sous la direction de Lavedan et, plus encore, après son départ en 1964 et son remplacement par le juriste Georges Burdeau, que cette marginalisation s'opère. La réforme des enseignements et le renforcement des autres sciences humaines au sein de l'IUUP dans les années 1950, ne laissent au cours d'histoire qu'un rôle restreint, presque exclusivement tourné vers l'histoire architecturale et des formes urbaines. La géogra-

6. Jean Gohier, Note manuscrite « Observations à l'intention de M. Ihrig sur son mémoire "Évolution de l'enseignement de l'urbanisme en France" », août 1967, Archives de Fontainebleau. 
phie et la sociologie prennent de l'importance avec Maximilien Sorre, Georges Chabot, René Clozier et Jean Margot-Duclos. Dès le milieu des années 1960, le cours d'histoire assuré par Hugueney et Picard s'efface au profit de la géographie qui devient dominante avec Philippe Pinchemel, Jacqueline Beaujeu-Garnier et Pierre George, tout comme la sociologie urbaine, alors en plein essor institutionnel avec Henri Lefebvre et Hubert Tonka. La volonté est de privilégier l'histoire la plus contemporaine. En 1967, il est ainsi prévu de faire appel à Robert Auzelle pour enseigner l'histoire de la Reconstruction, chaque enseignant se réservant dorénavant d'exposer l'histoire de son champ. Cette tendance s'accentue après 1968 et le déménagement en 1969 de l'iuUP à la Faculté de Dauphine. En 1971, le nouveau directeur Robert de Caumont, dans un éditorial de La vie urbaine, énumérant les disciplines motrices de la recherche en urbanisme ne mentionne pas l'histoire, discipline perçue comme classique, parcellaire et passéiste (Archives de P. Pinchemel). L'héritage de Lavedan et sa figure mandarinale sont contestés. Le personnage est moqué dans l'Institut autogéré de 1968. Les rapports de la Commission enseignement de l'IUUP, conservés dans les archives de Philippe Pinchemel, font ainsi souvent allusion à Lavedan, critiqué pour ses positions politiques et l'importance donnée à l'histoire au sein de l'Institut. Quand, enfin, l'Institut est reconnu, en 1969, comme unité d'enseignement et de recherche de $3^{\mathrm{e}}$ cycle universitaire, l'histoire est mise à l'écart, concurrencée par des disciplines plus en phase avec la réalité sociopolitique.

\section{L'HISTOIRE DANS LES THÈSES DE L'INSTITUT D'URBANISME de l'université de Paris}

L'analyse des façons dont l'histoire est mobilisée dans les mémoires de fin d'études de l'Institut, - dits aussi « thèses », selon l'usage à l'époque permet d'éclairer ces deux étapes. Rédigés par les étudiants juste avant leur sortie de l'IUUP sur un sujet libre, ils offrent un angle de vue privilégié sur ce qui est transmis et retenu de l'enseignement, les sujets relevant autant des tendances collectives - donc des jeux d'influence au sein du corps enseignant - , que des choix personnels et des contextes urbanistiques. C'est dire l'importance d'une telle série pour appréhender la formation d'une culture urbanistique, puisque ces étudiants occuperont, en France et à l'étranger, des postes à responsabilité dans le domaine de l'urbanisme. Ces thèses illustrent également l'évolution de la recherche française en urbanisme.

La quasi-totalité des thèses est conservée dans le fonds historique de l'IuP : on en dénombre 421 (de 1921 à 1969) auxquelles s'ajoutent celles de la période Dauphine (1969-1971), non encore inventoriées. Deux types de travaux font appel à l'histoire. Les thèses « d'évolution urbaine » à caractère monographique constituent l'exercice classique attendu de Marcel Poëte. Elles intègrent au plus une dimension prospective et aboutissent dans tous les 
cas à un regard sur le présent. Les thèses d' « histoire des villes » portent strictement sur le passé et ne se réfèrent pas nécessairement à une ville en particulier. La mise en graphique de la distribution de ces deux types de travaux dans la durée (figure 1) révèle deux grandes tendances : la forte baisse générale, des années 1920 aux années 1960, des travaux faisant appel à l'histoire, due essentiellement à celle des thèses d'évolution urbaine ; une croissance relative des thèses d'histoire des villes à proprement parler après la Seconde Guerre, puis leur diminution dans les années 1960.

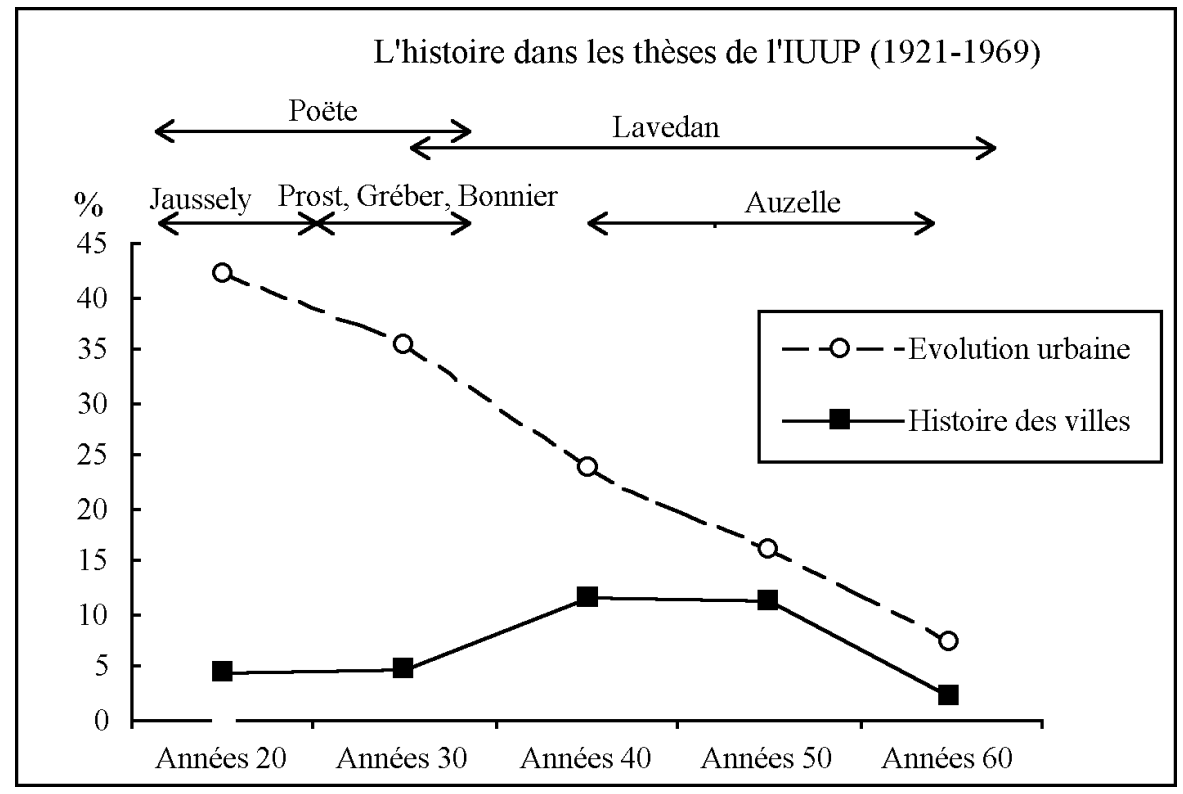

Fig. 1 - L'histoire dans les thèses de l'IUUP (1921-1969)

Au début de la période, les thèses faisant appel à l'histoire relèvent presque toutes de l'exercice d'évolution urbaine, le nombre des thèses portant strictement sur le passé étant très faible. Les titres mentionnent majoritairement le terrain de l'étude suivi des termes «étude d'évolution urbaine » ou «passé, présent, avenir », ce qui illustre le crédit de l'approche développée par Poëte. Du reste, l'exercice est également porté par d'autres enseignants, notamment les praticiens Louis Bonnier et Henri Prost. Mais le degré de compréhension des théories d'évolution urbaine exposées par Poëte est assez variable suivant les étudiants. Si certains font appel à l'histoire de façon plutôt formelle, d'autres, en particulier ceux dont le travail est mené sous la direction de Poëte témoignent d'une sensibilisation plus fine à ses propos et méthodes. Ils reflètent directement ses conceptions, tant sur le caractère organique de la ville, que sur la valeur heuristique de l'étude d'évolution urbaine dans la planification. Ainsi l'étudiant Kopp, dans sa thèse soutenue en 1934, écrit-il ces mots, très inspirés des 
approches de son directeur : « [L]'urbaniste ne pourra s'orienter dans la nuit du devenir que s'il y est aidé par la connaissance de la genèse des faits antérieurs, c'est à lui qu'appartiendra le soin de dégager l'esprit des formes et de discerner les relations essentielles entre les effets et les causes » (Kopp, 1934, 7). Avant guerre, les approches développées par Marcel Poëte, ou relayées par d'autres enseignants, passent bien auprès des étudiants, écho de la considération qui leur est accordée dans l'organisation pédagogique de l'Institut.

Bien que porté, à partir de la guerre, par une nouvelle équipe, l'architecte Pierre Rémaury, le géographe René Clozier et surtout Pierre Lavedan, l'exercice concerne encore, dans les années 1940 du moins, plus du quart des mémoires. Par la suite, la proportion devient mineure : les enseignants ayant entretenu la tradition sont amenés eux-mêmes, hormis René Clozier, à l'abandonner progressivement. L'exercice change de type : des monographies accordant une large place à l'évolution urbaine, on passe, à la fin des années 1940, à des monographies à visées planificatrices réduisant la part des études relatives au passé. Elles sont, dans certains cas, renvoyées explicitement " aux historiens » et constituent un travail de seconde main (Madurir de Aduiar, 1954), ce qui témoigne d'un fort éloignement, dans les représentations du moins, du travail d'aménageur d'avec celui de l'historien. Quant à Lavedan, dans un souci de continuité, il dirige lui aussi un certain nombre de monographies, mais ces dernières ne portent plus que sur le passé et le présent. La partie prévisionnelle sur l'avenir de la ville y est réduite au minimum, parfois une seule page en conclusion, pour disparaitre peu à peu et être remplacée par un inventaire des monuments hérités du passé à conserver. L'exercice d'évolution urbaine en est ainsi profondément modifié, tant dans ses méthodes, que dans ses finalités.

Simultanément à une baisse des thèses d'évolution urbaine, cette période est marquée par une légère croissance quantitative des thèses d'histoire portant spécifiquement sur le passé. L'origine de ce processus, dû quasi exclusivement à l'impulsion de Lavedan, remonte aux années 1930. Les thèses qu'il dirige relèvent de l'histoire de l'urbanisme et de l'art, comme Sarnarobriva, études d'urbanisme romain à Amiens (François Vasselle, 1955). Elles portent également de plus en plus sur les monuments historiques (Les portes de la ville sous Louis XIV, Béatrice Duleau, 1952). On y retrouve le souci de Lavedan de tirer parti des expériences du passé dans l'aménagement présent des villes, même quand l'analyse reste limitée au passé. Ainsi Jamusz Deryng, auteur de la première thèse d'histoire de l'Institut à traiter en grande partie du XIXe siècle, réalisée en 1951 sous la direction d'André Gutton, L'entourage de Notre-Dame de Paris, indique-t-il en introduction ${ }^{7}$ : «[Je]

7. Presque absente des thèses d'avant-guerre, cette période et les travaux d'Haussmann font peu à peu leur apparition dans les productions scientifiques de l'IUUP (thèses et La vie urbaine) et dans les cours de Lavedan, sous l'angle de la critique esthétique. 
voudrais étudier ce sujet, non comme un problème d'évolution historique, mais comme un problème contemporain et vivant. »

On enregistre néanmoins une lente diminution du nombre de thèses menées sous la direction de Lavedan, durant sa période d'activité (19401964), ce qui laisse supposer un affaiblissement de son autorité auprès des étudiants. Ce fait corrobore la marginalisation progressive de l'histoire à l'Institut, d'autant plus que les thèses d'histoire sont presque toutes dirigées par lui. La césure principale (baisse des thèses portant exclusivement sur le passé) se fait à la fin des années 1950. C'est le moment où l'histoire est concurrencée par les autres sciences humaines, entraînant une augmentation des thèses de géographie urbaine ou de sociologie, dirigées par René Clozier, Georges Chabot, Max Sorre. Ces dernières représentent en moyenne la moitié des thèses dans les années 1950 et plus encore dans les années 1960, avec Jacqueline Beaujeu-Garnier et Jean Margot-Duclos. Les géographes dirigent seuls, dès lors, les quelques thèses ayant recours à l'histoire, maintenant la flamme pour le passé des villes. Mais ces dernières disparaissent complètement après 1969 et le déménagement à Dauphine, où les diplômes ne portent plus que sur les propositions d'aménagement et les aspects politiques et sociaux de l'urbanisme.

Au total, les historiens de l'IUUP ont proposé deux conceptions de l'histoire urbaine. Poëte développe une approche vitaliste de la ville, là où Lavedan déploie une vision de la production des formes urbaines centrée sur les acteurs. Mais au-delà de leurs divergences, elles ont en commun de fournir un appareil théorique mobilisable pour l'observateur ou le praticien confronté au temps présent. Que l'on se réfère à l'interprétation de Poëte d'un cycle de vie urbain ou à la conception de Lavedan d'une histoire ressource dans la résolution des problèmes d'urbanisme, ces deux approches s'affranchissent de la sphère d'explication du seul passé pour proposer une interprétation processuelle du développement urbain (de la ville « cité » ou des formes urbaines).

Bien que complémentaires, ces deux approches de l'histoire urbaine ne se croisent pas : Pierre Lavedan impose son histoire de l'urbanisme et des formes urbaines au moment où les monographies de villes évolutionnistes inspirées par Marcel Poëte tendent à décliner. On peut faire l'hypothèse que l'éloignement progressif de Lavedan des théories de Poëte, dont il se veut au départ le successeur, constitue une tentative d'adaptation du discours historique au contexte de production urbaine de la Reconstruction et des « trente glorieuses ». Lavedan récupère en effet les critiques émises à l'encontre de Poëte en donnant son crédit à l'action de l'homme dans le développement urbain, position peut-être attendue de la part d'un historien d'art. Son approche se heurte toutefois à un climat intellectuel plus enclin à privilégier le présent. À l'Institut, l'histoire des villes de Lavedan est moins directement mobilisée par les urbanistes que ne l'est l'évolution urbaine de Marcel Poëte. 
Le parcours de l'histoire à l'iuUP révèle en somme les différentes acceptions accordées à l'urbanisme, compris au départ comme « science » et « art » urbain, bientôt écartelé entre l'" art » et la «science ». L'approche de Lavedan, à la différence de celle de Poëte, peine à se lier aux conceptions des praticiens de l'Institut. Toutes deux n'en restent pas moins tournées vers l'art urbain. Cela peut expliquer que l'histoire urbaine, prise au sens large, est nourrie aujourd'hui encore par une production provenant d'horizons disciplinaires différents, notamment celle des historiens ou encore celle des urbanistes eux-mêmes.

\section{Abréviations}

EHEU : École des hautes études urbaines (1919-1924)

IUUP : Institut d'urbanisme de l'université de Paris (1924-1971)

IUP : Institut d'urbanisme de Paris (1972)

PAEE : Plans d'aménagement, d'embellissement et d'extension

$\mathrm{SFU}$ : Société française des urbanistes

\section{RÉFÉRENCES BIBLIOGRAPHIQUES}

Archives de l'iuup : Comptes rendus du Comité de perfectionnement et du Conseil d'administration (vol. 1, 1919-1932 ; vol. 2, 1932-1967).

Archives de Philippe Pinchemel concernant la période 1967-1972.

Cours des enseignants de l'EHEU/IUUP (1919-1920, 1926-1927, 1937-1938, années 1950 et 1960).

Archives nationales : Programmes des cours de l'IUUP, AJ/16/8332.

AgAChe, D.-A. ; Auburtin, J.-M. ; REDONT, E. 1915. Comment reconstruire nos cités détruites. Notions d'urbanisme s'appliquant aux villes, bourgs et villages, Paris, Armand-Colin.

Auzelle, R. 1967. Cours d'urbanisme à l'Institut d'urbanisme de l'université de Paris. Première année. Intelligence du milieu et stratégie de l'aménagement, tome 1, Conférences liminaires par Robert Auzelle, Paris, Éditions Vincent Fréal et Cie.

BARDET, G. 1948 [1941]. Problèmes d'urbanisme, Paris, Dunod.

BAUDOUI, R. 1988a. La naissance de l'École des hautes études urbaines et le premier enseignement de l'urbanisme en France, des années 1910 aux années 1920, Paris, École d'architecture de Paris-Villemin/ARDU, Paris VIII.

Baudoui, R. 1988b. « Marcel Poëte et Le Corbusier : l'histoire dans le projet d'urbanisme », Les annales de la recherche urbaine, $\mathrm{n}^{\circ} 37$.

BRuANT, C. 2001. « Donat Alfred Agache (1875-1959). L'urbanisme, une sociologie appliquée », dans V. Berdoulay et P. Claval, Aux débuts de l'urbanisme français, Paris, L'Harmattan.

Busquet, G. ; Carriou, C. ; Coudroy de Lille, L. 2005. Un ancien institut... Une histoire de l'Institut d'urbanisme de Paris, Créteil, université de Paris XII, Valde-Marne.

CALABI, D. 1998, Marcel Poëte et le Paris des années vingt : aux origines de "l'histoire des villes », Paris, L'Harmattan. 
Claude, V. 2006. Faire la ville. Les métiers de l'urbanisme au XXe siècle, Marseille, Parenthèses, Eupalinos.

Deryng, J. 1951. L'entourage de Notre-Dame de Paris, sous la direction d'André Gutton, thèse $\mathrm{n}^{\circ} 275$.

GrÉBER, J. 1933. Ville de Marseille, plan d'aménagement et d'extension : mémoire descriptif. Paris, Fréal.

GRUdET, I. 2005a. L'Histoire de l'urbanisme de Pierre Lavedan de 1919 à 1955 : entre savoir et action, Doctorat de l'université de Paris VIII, Vincennes-Saint Denis.

GRUDET, I. 2005b. « De la transmission de connaissances à la diffusion de concepts opératoires. La relation entre le savoir et l'action étudiée à travers les figures de deux tomes de "L'Histoire de l'urbanisme" de Pierre Lavedan : "AntiquitéMoyen Âge" (1926) et "Période Contemporaine" (1952) », dans F. Pousin (sous la direction de), Figures de la ville et construction des savoirs, Paris, CNRS éditions, 2005, p. 164-172.

JAuSSEly, L. 1920. Art urbain, Cours de l'IuUP.

Kopp, M.-J. 1934. Laon. Sa vie, son évolution. Le passé. L'avenir, sous la direction de Marcel Poëte, thèse ${ }^{\circ} 92$.

LaVedAn, P. 1926, 1941, 1952. Histoire de l'urbanisme, Paris, H. Laurens : tome 1, Antiquité-Moyen Âge ; tome 2, Renaissance et Temps modernes ; tome 3, Époque contemporaine.

LaVEDan, P. 1959 [1937]. Géographie des villes, Paris, Gallimard.

LaVedan, P. 1993 [1975]. Histoire de l'urbanisme à Paris. Nouvelle histoire de Paris, Paris, Hachette.

LAVEDAN, P. 1919. La vie urbaine, $\mathrm{n}^{\circ} 1-2$.

LAVEDAN, P. 1933. La vie urbaine, $\mathrm{n}^{\circ} 17$.

LAVEDAN, P. 1953. La vie urbaine, $\mathrm{n}^{\circ} 3-4$.

MADURIR DE ADUIAR, M. 1954. Reconstruction à long terme. Étude d'un vieux quartier de Porto. Propositions de réaménagement, examen critique et méthode, sous la direction de Robert Auzelle, thèse $n^{\circ} 290$.

PAYRE, R. 2005, «Un savoir "utilitaire, scientifique et vulgarisateur" : la ville dans La vie urbaine, objet de science et objet de réforme (1919-1939) », dans Genèses $\mathrm{n}^{\circ}$ 60, «La ville des savants », Paris, Belin, p. 5-30.

PINON, P. 1996. « Pierre Lavedan, de l'histoire de l'art à l'architecture urbaine », dans Le visiteur. Ville, territoire, paysage, architecture, $\mathrm{n}^{\circ} 2$, Paris, SFA.

POËTE, M. 1919-1920, 1926-1927. Évolution des villes, Cours de l'IUUP.

Pö̈TE, M. 1929. Introduction à l'urbanisme. L'évolution des villes, la leçon de l'Antiquité, Paris, Boivin.

PuYo, J.-Y. 2001. « L'urbanisme selon Léon Jaussely », dans V. Berdoulay et P. Claval Aux débuts de l'urbanisme français, Paris, L'Harmattan.

RosenthaL, L. 1918. Villes et villages français après la guerre. Aménagement, restauration, embellissement et extension, préface de Louis Bonnier, Paris, Payot et Cie.

TONEFF, L. 1930. Ville de Varna. Principal port de Bulgarie à la mer noire. Étude de son état actuel expliqué par le passé. Son aménagement, embellissement et extension, sous la direction de Marcel Poëte, thèse $n^{\circ} 47$. 